\title{
Autocrine PDGFR signaling promotes mammary cancer metastasis
}

\author{
Martin Jechlinger, ${ }^{1}$ Andreas Sommer, ${ }^{1}$ Richard Moriggl, ${ }^{1}$ Peter Seither, ${ }^{2}$ Norbert Kraut, ${ }^{3}$ \\ Paola Capodiecci, 4 Michael Donovan, ${ }^{4}$ Carlos Cordon-Cardo, ${ }^{5}$ Hartmut Beug, ${ }^{1}$ and Stefan Grünert ${ }^{1}$
}

${ }^{1}$ Research Institute for Molecular Pathology, Vienna, Austria. ${ }^{2 B}$ Boehringer Ingelheim Pharma KG, Genomics Group, Biberach, Germany. ${ }^{3}$ Boehringer Ingelheim Austria GmbH, Department of Lead Discovery, Vienna, Austria. ${ }^{4}$ Aureon Laboratories, Yonkers, New York, USA. ${ }^{5}$ Memorial Sloan-Kettering Cancer Center, New York, New York, USA.

\begin{abstract}
Metastasis is the major cause of cancer morbidity, but strategies for direct interference with invasion processes are lacking. Dedifferentiated, late-stage tumor cells secrete multiple factors that represent attractive targets for therapeutic intervention. Here we show that metastatic potential of oncogenic mammary epithelial cells requires an autocrine PDGF/PDGFR loop, which is established as a consequence of TGF- $\beta$-induced epithelialmesenchymal transition (EMT), a faithful in vitro correlate of metastasis. The cooperation of autocrine PDGFR signaling with oncogenic Ras hyperactivates PI3K and is required for survival during EMT. Autocrine PDGFR signaling also contributes to maintenance of EMT, possibly through activation of STAT1 and other distinct pathways. Inhibition of PDGFR signaling interfered with EMT and caused apoptosis in murine and human mammary carcinoma cell lines. Consequently, overexpression of a dominant-negative PDGFR or application of the established cancer drug STI571 interfered with experimental metastasis in mice. Similarly, in mouse mammary tumor virus-Neu (MMTV-Neu) transgenic mice, TGF- $\beta$ enhanced metastasis of mammary tumors, induced EMT, and elevated PDGFR signaling. Finally, expression of PDGFR $\alpha$ and $-\beta$ correlated with invasive behavior in human mammary carcinomas. Thus, autocrine PDGFR signaling plays an essential role during cancer progression, suggesting a novel application of STI571 to therapeutically interfere with metastasis.
\end{abstract}

\section{Introduction}

Carcinomas account for more than $80 \%$ of human cancers, generated through a multistep process (1) eventually leading to metastasis, the major cause of cancer morbidity. In normal epithelia, homeostasis, differentiation, and organ integrity are regulated by interactions among epithelial cells, the extracellular matrix, and adjacent nonepithelial cells. Normal epithelial cells cannot digest and migrate through the basement membrane (2) and undergo apoptosis upon displacement from their substratum (anoikis; ref. 3). During tumor progression, cancer cells gain anchorage independence and the ability to transmigrate tissues, a prerequisite for metastasis to distant sites $(4,5)$. Multiple molecular players contribute to the acquisition of invasive properties by tumor cells $(1,6)$, but we are only beginning to understand the molecular mechanisms involved.

One of the key players in late-stage tumor progression is TGF- $\beta$ $(7,8)$, which promotes metastasis in several murine tumor models (9-13). Loss of TGF- $\beta$ receptor II (TGF- $\beta$ RII) also correlates with good prognosis in colon carcinoma patients with DNA mismatch repair deficiency (microsatellite instability or MIN type; ref. 14). Likewise, acquisition of metastatic potential in skin tumors is associated with activation of TGF- $\beta$ R signaling through loss of the natural TGF- $\beta$ R decoy receptor NMA/Bambi (15). In normal epithelial cells, TGF- $\beta$ causes cell-cycle arrest and apoptosis, which contributes to the tumor-suppressive function of TGF- $\beta$ R signaling in early stages of tumorigenesis $(7,9)$. During late-stage tumor

Nonstandard abbreviations used: dnP, dominant-negative PDGFR; EMT, epithelial-mesenchymal transition; EpRas cell, Ras-transformed EpH4 cell; FDCP-1, factordependent continuous cell line, Paterson Laboratories 1; MMTV, mouse mammary tumor virus; RTK, receptor tyrosine kinase; TGF- $\beta$ RII, TGF- $\beta$ receptor II. Conflict of interest: The authors have declared that no conflict of interest exists. Citation for this article: J. Clin. Invest. 116:1561-1570 (2006). doi:10.1172/JCI24652. progression, this antiproliferative response to TGF- $\beta$ is lost, for instance, through inactivating mutations in TGF- $\beta$ RII (16) and Smad4 (17) in human colon and pancreatic carcinomas, respectively. In most tumor types, including mammary carcinomas, however, mutations in TGF- $\beta$ R or Smads are rare, and tumor cells commonly abolish the antiproliferative function of TGF- $\beta$ while remaining responsive to the factor. This may occur through loss/mutation of TGF- $\beta$-regulated tumor suppressors (such as p $15^{\mathrm{INK} 4 \mathrm{~B}}$; ref. 18 ) and/or cooperation with hyperactive Ras $(7,19)$.

Consistent with the ability of oncogenic Ras to overcome the TGF- $\beta$-mediated proliferation arrest, receptor tyrosine kinases (RTKs; ref. 20), and Ras family proteins $(21,22)$ are mutated, amplified, or hyperactivated in many human cancers. Furthermore, in several epithelial cell models, including the murine mammary epithelial cell line EpH4, metastatic potential requires the cooperation of TGF- $\beta$ signaling with hyperactive ERK/MAPK signaling, caused by oncogenic RTKs (e.g., HER2), Ras, or Raf (23-26). In Ras-transformed EpH4 cells (EpRas cells), metastatic potential strictly correlated with the ability to undergo TGF- $\beta$-induced epithelial-mesenchymal transition (EMT) $(27,28)$. EpH4 cells that are protected from apoptosis but lack Ras-dependent MAPK hyperactivation are devoid of metastatic potential and fail to undergo persistent changes in epithelial marker gene expression $(19,28)$. Rather, these cells display a migratory phenotype in response to TGF- $\beta$, which is distinct from EMT and was termed "scattering."

As originally defined (2), EMT involves transdifferentiation of epithelial cells to fibroblastoids, invasive cells with the ability to transmigrate the basal lamina, showing a profoundly altered, mesenchymal gene expression program $(19,29)$. Recently, multiple developmental signaling pathways and transcription factors (Wnt, Notch, Hegdehog, NF-кB, Snail superfamily, Twist) were shown to contribute to EMT and promote tumor cell invasion at the same time (reviewed in 


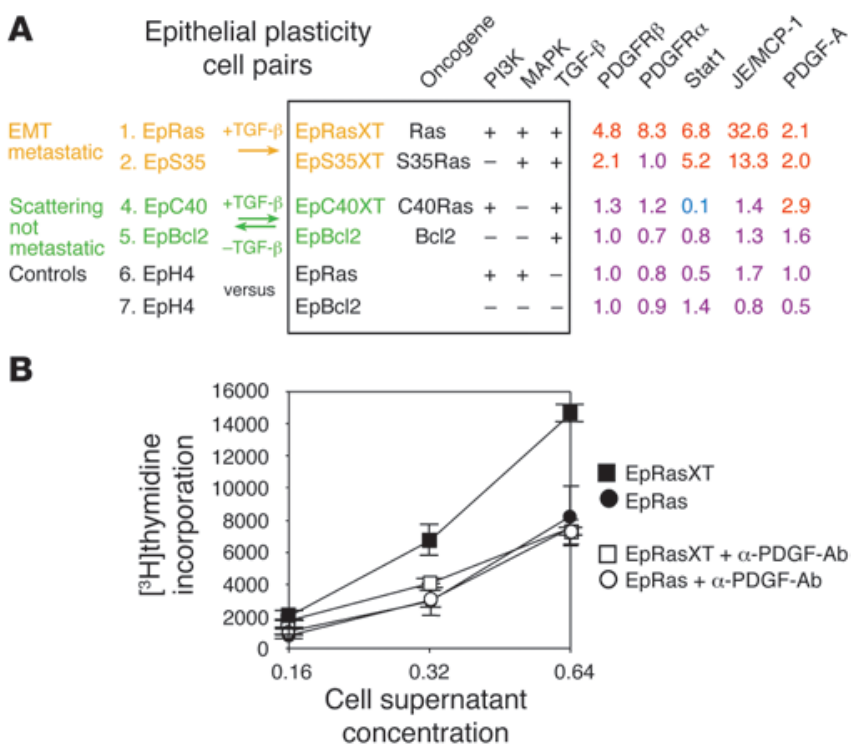

refs. 30, 31). In many human carcinomas, however, detection of EMT can be difficult due to its frequently transient nature (32).

Recently we employed expression profiling to find genes selectively upregulated during EMT, identifying multiple genes upregulated during late-stage tumor progression (29). This metastasis-specific gene set included PDGF, components of the PDGFR signaling pathway, and known target genes (29), suggesting establishment of an autocrine PDGF/PDGFR loop in an EMT-specific fashion. So far, autocrine PDGF signaling has almost exclusively been described in nonepithelial tumors, e.g., gliomas (33). While expression of PDGF correlates with advanced tumor stages and unfavorable prognosis in human breast carcinomas $(34,35)$, PDGF produced in carcinomas is generally thought to act on the nonepithelial tumor stroma, for instance, by promoting angiogenesis $(36,37)$.

Here, we show that an autocrine PDGF signaling loop coincides with TGF- $\beta$-induced EMT in mammary epithelial cells and mammary tumors in mouse mammary tumor virus-Neu (MMTV-Neu/

\section{Figure 2}

Increased metastasis induced by RTK plus TGF- $\beta$ signaling in a transgenic mammary carcinoma model correlates with EMT and invasion. (A) Spontaneous formation of lung metastases in transgenic mice bearing a normal (MMTV-Neu) or constitutively active (MMTV-C.A.-Neu) EGFR 2 (HER2) under the control of the MMTV promoter (40) before or after breeding MMTV-TGF- $\beta 1$ transgenic mice (Neu/TGF- $\beta$, C.A.-Neu/TGF- $\beta$ ). Thirty to 36 mice per group were analyzed for metastasis formation, and differences in numbers of metastases between the different groups were significant $(P<0.01)$. (B) Primary tumors from MMTV-Neu mice or MMTV-Neu $\times$ MMTV-TGF- $\beta 1$ mice were subjected to cryosectioning and histological staining. (C) Cryosections from the above-described tumors were stained with antibodies against vimentin (red, left panels) or tenascin C (TN-C; green) plus CD31 to indicate endothelial cells (red; see Methods). DNA (nuclei) stained with DAPI (blue). MMTV-Neu tumors expressed tenascin C only in CD31-positive endothelial cells from tumor blood vessels, while the MMTV-Neu $\times$ MMTV-TGF- $\beta 1$ tumors clearly expressed tenascin $C$ in the tumor cells themselves. Original magnification, $\times 40$ (B and C). (D) Tumors from MMTV-Neu or MMTV-Neu $\times$ MMTV-TGF- $\beta$ mice were processed for mRNA extraction and realtime PCR, using oligonucleotide primers for PDGF-A, PDGF-B, and the PDGFR target gene JE/MCP-1. The amount of mRNA obtained for double transgenic tumors was higher in all cases $(P<0.05)$.

\section{Figure 1}

EMT-specific upregulation of PDGFR pathway genes generates an autocrine PDGF/PDGFR loop. (A) Regulation of PDGFR pathway genes in various cell pairs based on $\mathrm{EpH} 4$ mouse mammary epithelial cells that undergo TGF- $\beta$-induced alterations in epithelial plasticity (see ref. 29). Two cell pairs underwent EMT in response to TGF- $\beta$ (yellow), 2 underwent reversible scattering (green), and 2 cell pairs served as oncogene controls (black). Shown are the oncogenes cooperating with TGF- $\beta$, the predominant signaling pathways activated, and the fold regulation of 5 PDGF pathway genes (red: upregulation; pink: no significant regulation, blue: downregulation). (B) Concentrated, serum-free supernatants from EpRas and EpRasXT cells were tested on huPDGFR-A/FDCP- 1 cells for mitogenic activity ( $\left.{ }^{3} \mathrm{H}\right]$ thymidine incorporation). Specificity of the mitogenic response for PDGF was verified by addition of saturating amounts of a neutralizing $\alpha-P D G F-A / B$ antibody.

TGF- $\beta$ ) transgenic mice. Autocrine PDGFR signaling essentially contributes to EMT, since its blockade abolishes TGF- $\beta$-induced EMT in EpRas cells, inducing apoptosis instead. This is due to interference with PDGFR-dependent hyperactivation of PI3K - the major signaling target of the PDGFR in vivo $(38,39)$ - which is required for apoptosis protection during EMT (24). Furthermore, interference with PDGF signaling abolished the established mesenchymal phenotype in EpRasXT cells and caused apoptosis in human mammary carcinoma cell lines. These findings are of physiological relevance, since inhibition of PDGF signaling by STI571 or expression of a dominant-negative PDGFR (dnP) strongly reduced the metastatic potential of EpRas cells in an in vivo metastasis assay.

A

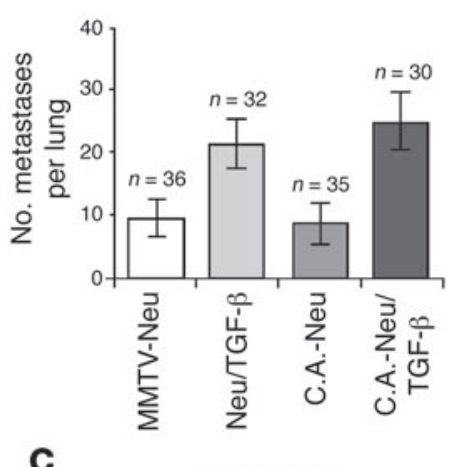

B

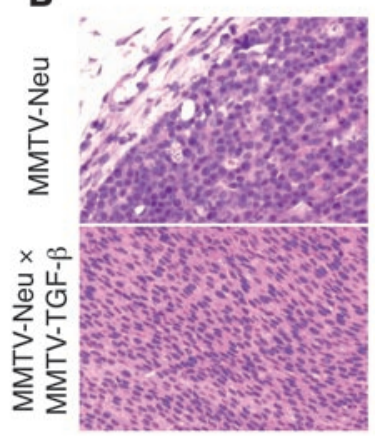

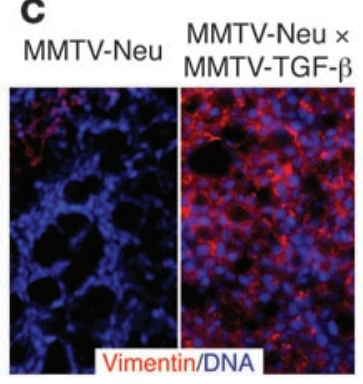

D

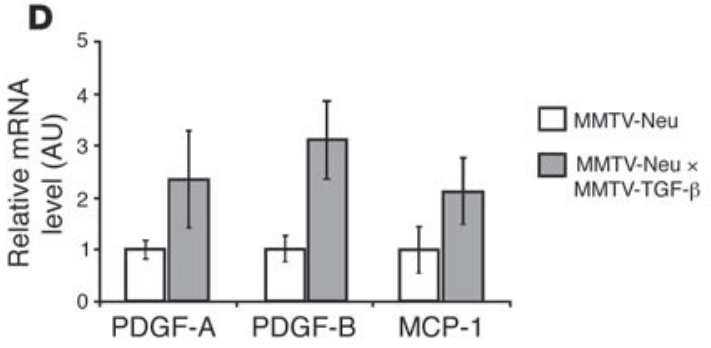




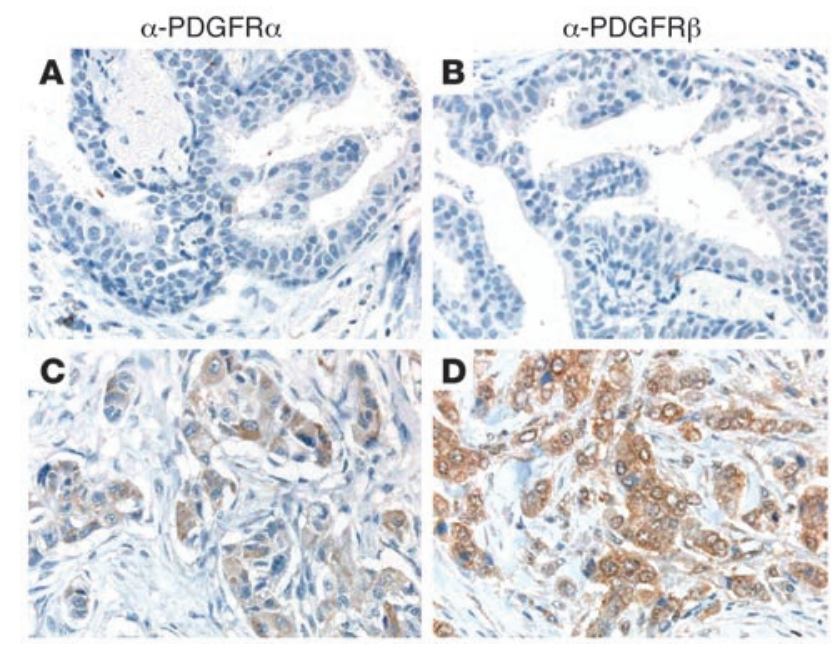

Finally, expression of both PDGFR $\alpha$ and $-\beta$ was not detectable in normal human mammary tissue but correlated with an invasive phenotype in primary human mammary carcinomas. Our results thus establish a cell-autonomous, functional role of the PDGFR during metastasis of mammary carcinoma cells, suggesting the PDGFR as a potential target for pharmacological intervention to reduce the metastatic potential of breast cancer.

\section{Results}

TGF- $\beta$ induces an autocrine PDGF loop during EMT. Using comparative expression profiling of $7 \mathrm{EpH} 4-\mathrm{based}$ cell pairs undergoing EMT, scattering, or oncogenic transformation, PDGFR signaling was found to be upregulated exclusively during EMT (Figure 1A; ref. 29; for a detailed description of cell types used, see Supplemental Methods; Supplemental material available online with this article; doi:10.1172/JCI24652DS1). In mesenchymal EpRasXT cells, increased expression of PDGF-A, PDGFR $\alpha$ and $-\beta$, and STAT1 and -2 chemokines (JE/MCP-1 and KC/Gro-1; known as PDGFR target genes) was observed, as compared with their epithelial counterparts. To verify that the PDGF induced during EMT was secreted and bioactive, we employed murine hematopoietic cells that express the human PDGFR $\alpha$ (huPDGFR/factor-dependent continuous cell line, Paterson Laboratories 1 [huPDGFR/FDCP-1] cells) and thus proliferate in a PDGF-dependent fashion. Concentrated, serum-free supernatants from EpRasXT but not EpRas cells stimulated proliferation of PDGFR-FDCP cells in a concentration-dependent fashion (Figure 1B). Saturating amounts of a PDGF-neutralizing antibody added to EpRasXT supernatants reduced this proliferation to background levels (Figure 1B), con-

\section{Figure 3}

PDGFR $\alpha$ and PDGFR $\beta$ expression is specifically upregulated in human late stage mammary tumors. (A and $\mathbf{B}$ ) Shown are typical examples of intraductal mammary carcinomas, in which no staining for PDGFR $\alpha$ (A) or PDGFR $\beta$ (B) could be detected. (C and D) Typical examples of invasive mammary carcinomas that show intense staining for PDGFR $\alpha$ (C) or PDGFR $\beta$ (D). Original magnification, $\times 40$ (A-D).

firming that bioactive PDGF is the active agent in these supernatants. Undiluted EpRasXT cell-conditioned medium contained 3.7-4.9 ng/ml PDGF - known to maximally stimulate proliferation of primary human fibroblasts - as determined by dilutions of conditioned medium compared with a standard solution of human recombinant PDGF-A.

Increased PDGFR signaling is correlated with EMT in mouse tumors models. To determine whether TGF- $\beta$ induces PDGFR signaling in vivo, we employed transgenic mice expressing the oncogenic RTK ErbB-2/Neu in the mammary gland under the control of the MMTV promoter. These mice form spontaneous mammary tumors with a weak tendency to progress to lung metastases (40). Crossing these mice with MMTV-TGF- $\beta 1$ mice delayed the onset of mammary tumors but strongly increased metastasis formation (Figure 2A; refs. 11, 41). MMTV-Neu $\times$ MMTV-TGF- $\beta$ tumor cells appeared to be more elongated as compared with the MMTV-Neu tumors (Figure 2B). Furthermore, MMTV-Neu $\times$ MMTV-TGF- $\beta$ tumor cells displayed upregulation of EMT markers such as vimentin and tenascin $\mathrm{C}$ (Figure 2C; ref. 29). Interestingly, these tumors also showed enhanced expression of PDGF-A, PDGF-B, and the PDGFR-signaling target JE/ MCP-1 (Figure 2D). In conclusion, spontaneous murine mammary carcinomas that express TGF- $\beta 1$ are highly metastatic and display aspects of EMT, including enhanced, autocrine PDGF signaling.

Consequently we aimed to extend our findings to human tumors and proceeded to test expression of PDGFR $\alpha$ and PDGFR $\beta$ by immunohistochemistry in intraductal and/or invasive mammary carcinomas and compared levels of expression with those in normal human mammary tissue. While epithelial cells lining normal ducts and acini showed undetectable levels of expression in all samples tested (all normal breast tissue stained; Figure 3, A and B), a large number of breast tumors displayed a positive PDGFR phenotype (Table 1). Invasive carcinomas expressed PDGFR $\alpha$ in $65 \%$ and PDGFR $\beta$ in $75 \%$ of samples; moreover, a considerable fraction of these tumors showed intense staining for both PDGFR $\alpha$ and $-\beta(17.2 \%$ and $25.8 \%$; Figure $3, \mathrm{C}$ and D, and Table 1). Together with our findings in mouse tumor models, these data are consistent with an important function for PDGFR signaling in breast tumor progression.

Autocrine PDGFR signaling: EMT-specific activation of the PI3K and STAT1 pathways. The PI3K pathway is the major target of PDGFR signaling in vivo $(38,39)$. We therefore addressed whether autocrine

\section{Table 1}

Analysis of 16 normal mammary tissues, 45 intraductal carcinomas, and 93 invasive carcinomas for their anti-PDGFR $\alpha$ or anti-PDGFR $\beta$ immunostaining levels

\begin{tabular}{|c|c|c|c|c|c|c|}
\hline & \multicolumn{3}{|c|}{ Anti-PDGFR $\alpha$ staining } & \multicolumn{3}{|c|}{ Anti-PDGFR $\beta$ staining } \\
\hline & Undetectable & Low to moderate & Intense & Undetectable & Low to moderate & Intense \\
\hline Normal breast, no. (\%) & $16(100)$ & 0 & 0 & $16(100)$ & 0 & 0 \\
\hline Intraductal carcinoma, no. (\%) & $32(71.1)$ & $13(28.9)$ & 0 & $24(53.3)$ & $21(46.7)$ & 0 \\
\hline Invasive carcinoma, no. (\%) & $32(34.3)$ & $45(48.4)$ & $16(17.2)$ & $32(24.7)$ & $45(49.5)$ & $16(25.8)$ \\
\hline
\end{tabular}

Staining was classified as undetectable (as shown in Figure 3, A and B), low to moderate, or intense (as shown in Figure 3, C and D). 
A

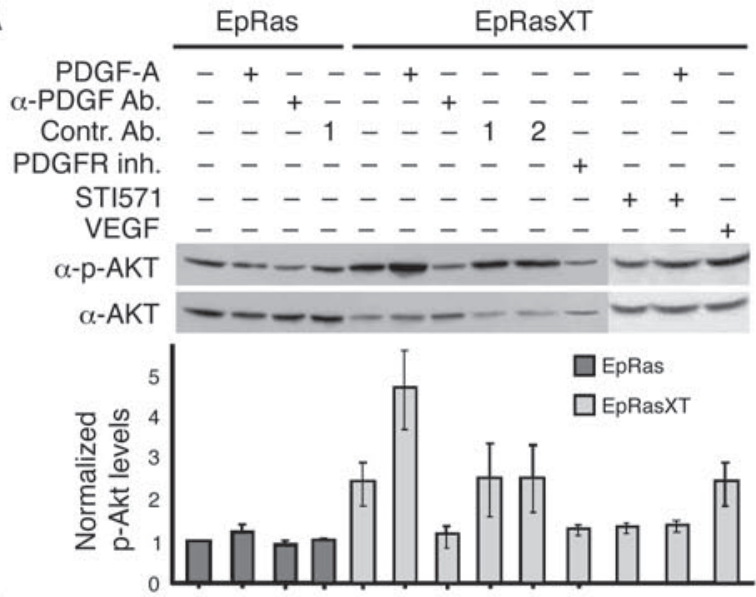

B
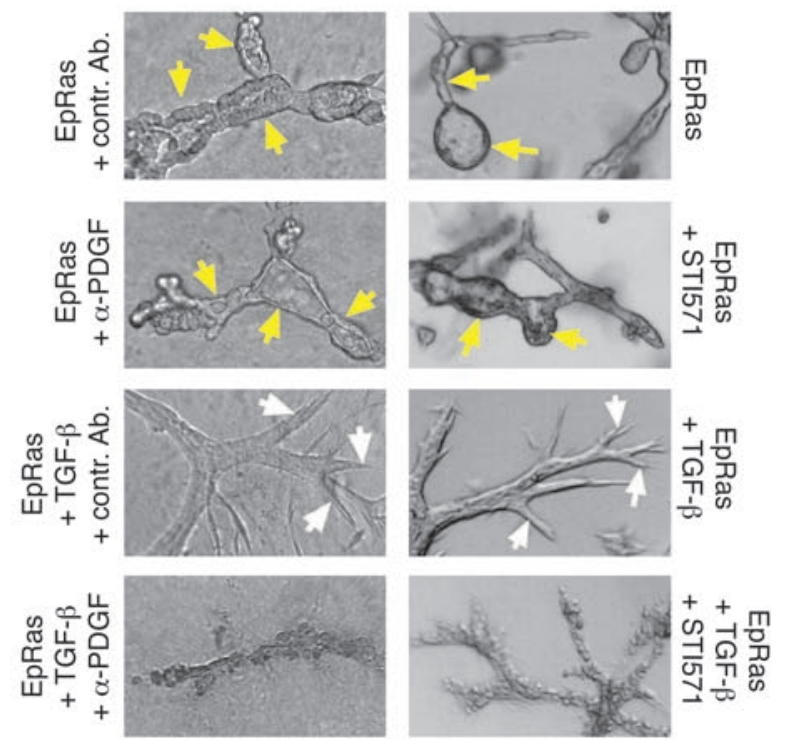

C
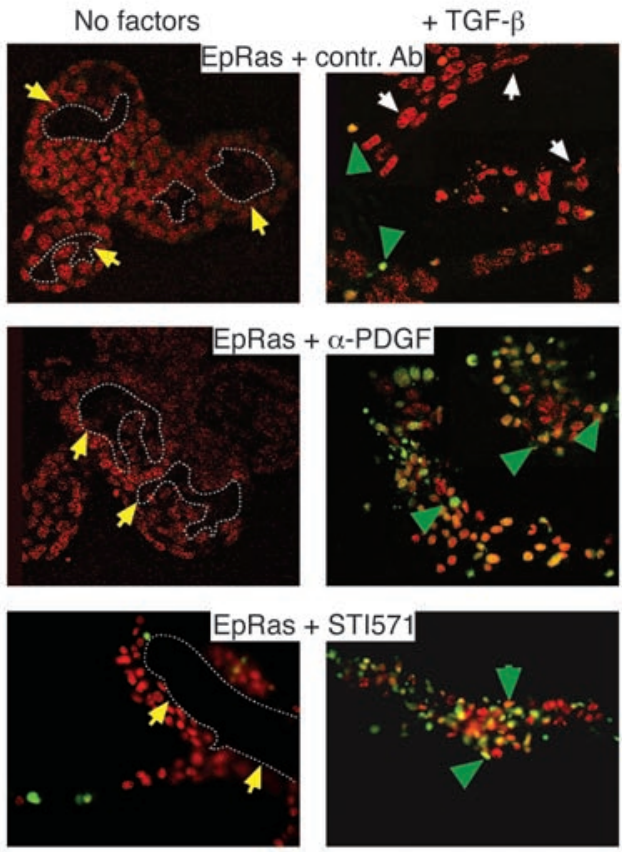

D

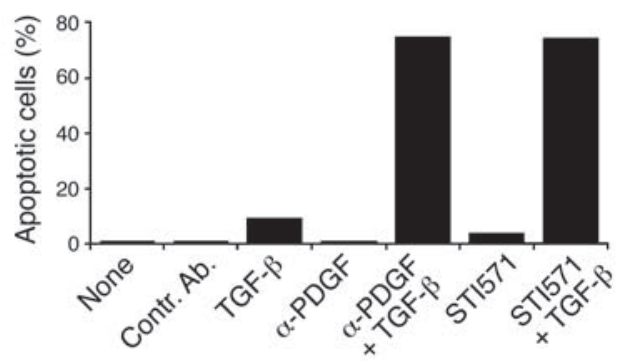

Figure 4

Interference with autocrine PDGF signaling in EpRas cells prevents EMT by causing apoptosis. (A) EpRas and EpRasXT cells were treated with PDGF, VEGF (control, VEGFR pathway not altered in EpRasXT cells), PDGF-neutralizing antibodies ( $\alpha$-PDGF-Ab), 2 nonimmune control antibodies (Contr. Ab 1 and 2), the tumor drug STI571, or a specific PDGFR tyrosine kinase inhibitor (PDGFR inh; see Methods). Levels of p-AKT were determined by Western blot analysis. For normalization, blots were stripped and reprobed for total AKT. Signals were quantified by densitometry, normalized to levels of untreated EpRas cells, and shown as histograms. Data from 3 independent experiments are represented as mean \pm SD. (B) EpRas cells were seeded into collagen gels and induced or not induced to undergo EMT by addition of TGF- $\beta$, in the presence or absence of neutralizing PDGF antibodies ( $\alpha$-PDGF), no immune antibodies (Contr. Ab), or STI571. Cultures were photographed after 7 days. (C) Similar collagen cultures were subjected to in situ TUNEL staining (green) and counterstaining for DNA (red). Lumina of polarized epithelial structures (yellow arrows), spindle-shaped mesenchymal cells (white arrows), and TUNEL-positive nuclei (green arrows) are indicated. Original magnification, $\times 10$ (B) and $\times 40$ (C). (D) Quantification of the data in B (>300 cells from several gel structures were evaluated for TUNEL and DAPI staining).

PDGF signaling in EpXT cells would enhance PI3K pathway activity by measuring p-AKT levels. In epithelial EpRas cells, neither PDGF nor PDGF-neutralizing antibodies altered p-AKT levels (Figure 4A). In contrast, EpRasXT cells showed a 2.5-fold higher PI3K activity than EpRas cells, which was further enhanced 2-fold by addition of PDGF (Figure 4A). Treatment with neutralizing PDGF antibody reduced PI3K activity in EpRasXT cells to the level of EpRas cells, while nonimmune antibodies failed to affect PI3K activity. Similar results were obtained with a specific inhibitor of the PDGFR tyrosine kinase and with the approved cancer drug STI571 (Gleevec), which inhibits several RTKs, including the PDGFR (42). PDGF also activated STAT1 in EpRasXT cells (39) (Supplemental Figure 1, A and B), which is known to contribute to breast cancer $(39,43)$. Indeed, both STAT1 expression and its activation by PDGF occur exclusively in cells able to undergo EMT (Supplemental Figure 1C), consistent with a possible role of STAT1 during tumor progression. In contrast to activating the PI3K and STAT1 pathway, PDGF had no reproducible effect on ERK/MAPK activity in either EpRas or EpRasXT cells (data not shown).

Autocrine PDGF/PDGFR signaling is required for apoptosis protection during EMT. PI3K activity protects EpRas cells from apoptosis during TGF- $\beta$-dependent induced EMT (19). Since PDGF signaling 
A
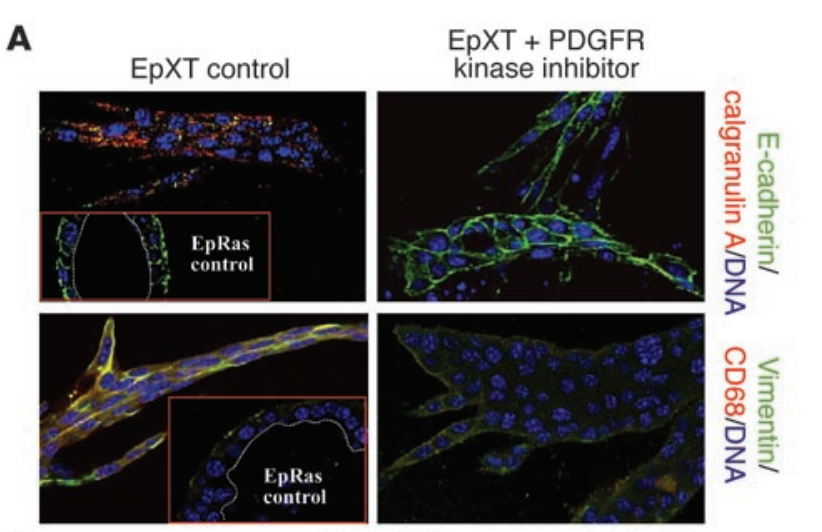

B

EpRasXT
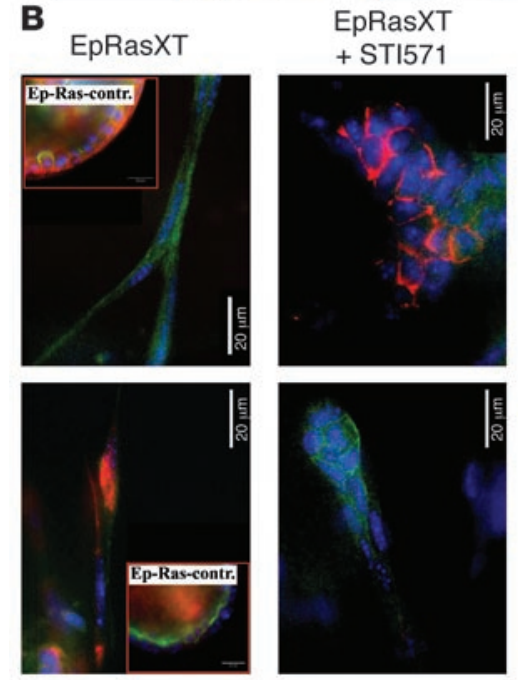

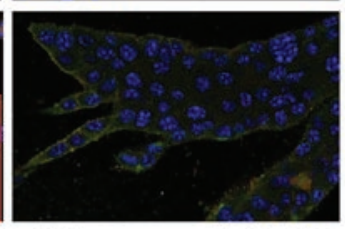

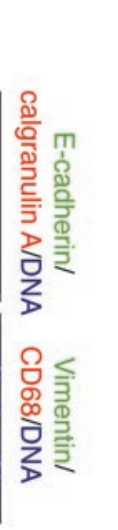

\section{Figure 5}

Interference with autocrine PDGF signaling in mesenchymal EpRasXT cells causes reversal of EMT rather than apoptosis. (A) EpRasXT cells were allowed to grow in collagen gels for 4 days and treated when indicated with the specific PDGFR tyrosine kinase inhibitor (see Figure 4) for another 5 days. Gels were stained for E-cadherin (green, top panels) and mesenchymal markers calgranulin A (red, top panels), vimentin (green, bottom panels), and CD68 (red, bottom panels). Insets: EpRas control structures stained with the same antibodies. (B) EpRasXT and EpRas cells (insets) were inhibitor-treated and stained for E-cadherin/ F-actin (top panels) or vimentin/F-actin (bottom panels), before (left) or after treatment with STI571 (middle) or the specific PI3K inhibitor LY294.002 (right). Original magnification, $\times 60$ (A) and ×100 (B).

completion of EMT require the high PI3K activity induced by autocrine PDGF signaling for viability, EpRasXT-derived mesenchymal structures were treated with the PI3K inhibitor LY294.002, at concentrations known to reduce PI3K signaling to basal levels typical for EpRas cells (44). LY294.002 affected neither cell viability nor the mesenchymal phenotype of the cells, which maintained vimentin expression and failed to express membrane-localized E-cadherin. (Figure 5B). These results indicate that autocrine PDGFR signaling is required for maintenance of EMT, but not for survival of cells that have already undergone EMT.

PDGFR signaling is required for EMT in vivo and metastatic potential of EpRas cells. Since TGF- $\beta$-induced EMT is closely correlated to metastatic ability in EpRas cells (19) and murine tumor models (Figure 2 and refs. 11, 12), we addressed whether autocrine PDGFR signaling is also necessary for metastatic potential in vivo. For this, we generated EpRas cell clones stably expressing a dnP tagged with GFP (EpRas-dnP; Figure 6A) (45). These EpRas-dnP clones formed epithelial structures indistinguishable from those formed by parental EpRas cells, both by morphology and epithelial marker expression in collagen gels (Figure 6B). TGF- $\beta$, however, failed to induce EMT in these EpRas-dnP clones, in contrast to EpRas control cells, which underwent EMT as expected (Figure 6B). Instead, the EpRas-dnP cells formed compact structures in response to TGF- $\beta$ that expressed membrane-bound, delocalized E-cadherin but no vimentin (Figure 6B), clearly resembling EpRasXT cells treated with PDGFR inhibitors (Figure 5).

EpRas-dnP cells and EpRas cells expressing GFP were injected into the mammary fat pad of nude mice, leading to tumor formation with identical incidence (data not shown) but clearly reduced tumor size (Figure 6C). Since hyperactive PI3K contributes to fast tumor growth (44), smaller tumors could be due to dnP-mediated reduced PI3K pathway activity. More detailed analysis of the tumors showed that EpRas-dnP tumors were more compact than EpRas control tumors, the tumor cells showing a less spindle-like morphology (Supplemental Figure 3). Furthermore, the (>90\% cytokeratin-positive) EpRas-dnP tumor cells retained delocalized E-cadherin expression and lacked vimentin, while EpRas control tumor cells were vimentin positive but essentially lacked E-cadherin expression (Supplemental Figure 2). In conclusion, dnP expression prevents EMT in EpRas cells both in collagen gels and mouse tumors (for details, see Supplemental Figures 2 and 3 ).

The metastatic potential of EpRas-dnPs and EpRas cells was assayed by tail vein injection. EpRas cell-injected mice succumbed to massive lung metastases, while mice receiving EpRas-dnP cells appeared healthy even after all control animals had died. They showed no increased lung weight and revealed only rare metasta- 
A
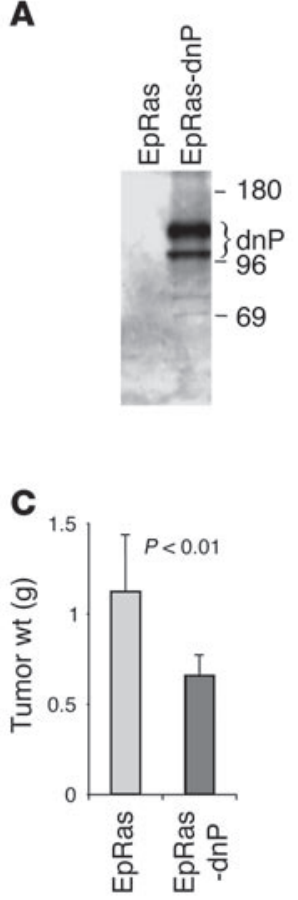

B
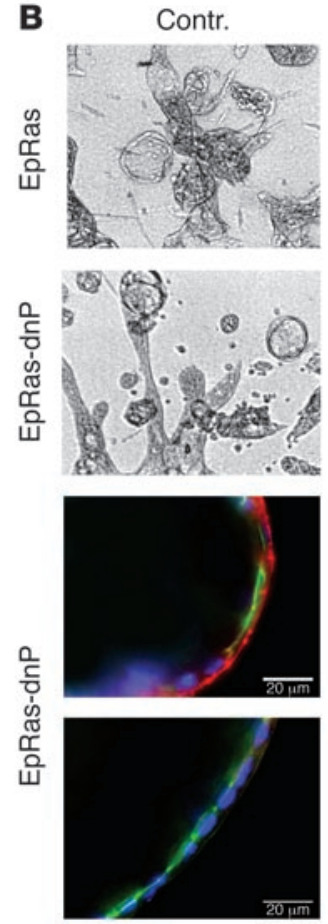

TGF- $\beta, 6 \mathrm{~d}$
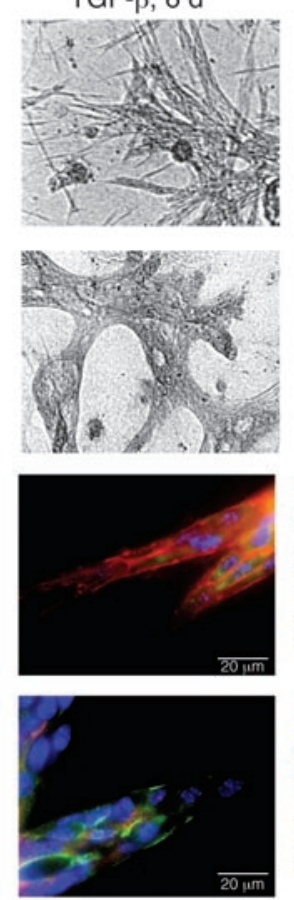

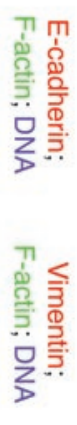

\section{Figure 6}

dnP expressed in EpRas cells prevents EMT but not tumor growth. (A) EpRas clones infected with retroviral vectors expressing dnP-GFP (EpRas-dnP) or GFP alone (EpRas) were analyzed for expression of exogenous dnP by Western blot analysis. (B) EpRas and EpRas-dnP cells were seeded into collagen gels and treated as indicated. Results of brightfield microscopy (top panels) and staining with $\alpha$-E-cadherin, $\alpha$-vimentin, and $\alpha-F$-actin antibodies (bottom panels) are shown. Original magnification, $\times 10$ (B, top panels) and $\times 60$ (B, bottom panels). (C) Cells as analyzed in A and B were injected into the fat pads of nude mice ( 6 mice per cell type; 2 injection sites per mouse; $2 \times 10^{5}$ cells per injection site) and total tumor weight determined after 2-3 weeks.

ses upon histological analysis (average: 1.8 metastases per lung), compared with an average of 142.5 metastases per lung in EpRasinjected animals (Figure 7, A and B).

To determine whether the observed inhibition of metastasis by the $\mathrm{dnP}$ is cell autonomous, we injected nude mice with mixtures of unlabeled EpRas cells and the same cells expressing either dnPGFP or empty GFP vectors. After 3 weeks, large lung metastases had developed in both groups of animals. However, cultured cells obtained from metastatic lungs of EpRas/EpRas-GFP-dnPinjected mice almost completely lacked GFP-positive cells (Figure 7C), while respective cells from control mice (EpRas/EpRasGFP mixture) contained 10-40\% GFP-positive cells (Figure 7C). Thus, EpRas-dnP cells even fail to contribute to lung metastases formed by neighboring EpRas cells, showing that PDGFR signaling contributes to the metastatic potential of EpRas cells in a cell-autonomous fashion.

STI571 suppresses metastasis in tumor cells employing autocrine PDGF signaling. The effects of an overexpressed $\mathrm{dnP}$ on endogenous PDGFR signaling might differ from those caused by neutralizing antibodies or tyrosine kinase inhibitors. We therefore employed STI571, which effectively blocked PDGFR signaling in our cells (Figures 4 and 5) and inhibits PDGFR tyrosine kinase activity in vivo after oral administration (37). Accordingly, doses of STI571 similar to those used in humans $(100 \mathrm{mg} / \mathrm{kg})$ were fed to nude
To address the significance of the above results in human cells, we tested 2 dedifferentiated, invasive human mammary carcinoma cell lines (MDA-MB231, CAMA-1; ref. 47; see Supplemental Figure 4) for their response to STI571 or PDGFR inhibitor. As expected, STI571 induced apoptosis in both human carcinoma lines at concentrations fully tolerated by CT26 control cells (Supplemental Figure 4B). A specific PDGFR inhibitor abolished the migratory phenotype in CAMA-1 cells, inducing compact structures instead (Supplemental Figure 4A).

\section{Discussion}

Oncogenes that hyperactivate MAPK and PI3K pathways are key players during tumor progression $(19,44)$. One of the major activities of these pathways is to overcome the proapoptotic and antiproliferative activities of TGF- $\beta$, which epithelial cells are subject to $(7,8,48,49)$. These oncogene-driven signaling pathways also promote local invasion and metastasis, in cooperation with TGF- $\beta$ signaling $(28,30)$. In this article, we show that (a) oncogenic Ras alone is insufficient to hyperactivate the PI3K pathway but requires cooperation with PDGF signaling to efficiently protect mammary epithelial cells from TGF- $\beta$-induced apoptosis during EMT; (b) autocrine PDGFR signaling is required for metastasis of Ras-transformed mammary epithelial cells in a cell-autonomous fashion; (c) pathways 
A
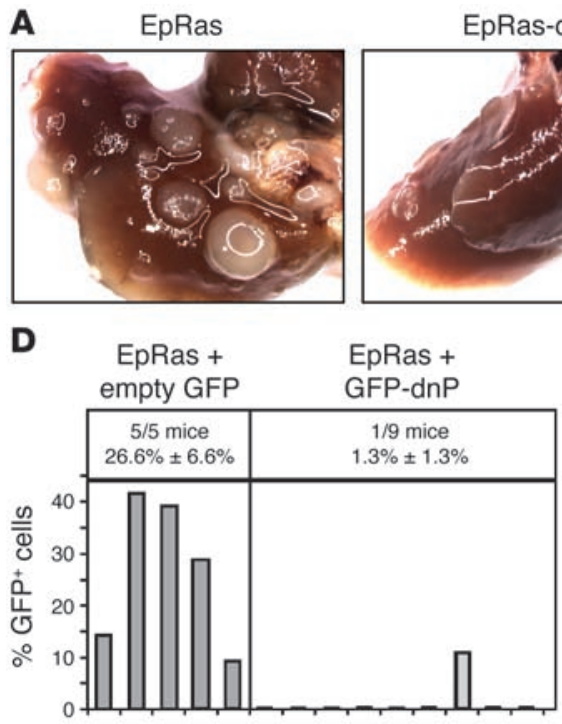

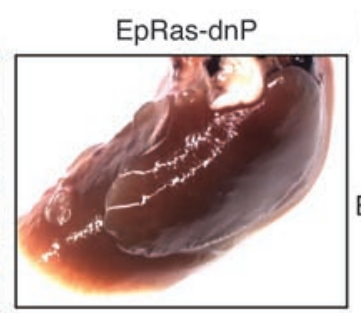

$\mathbf{E}$

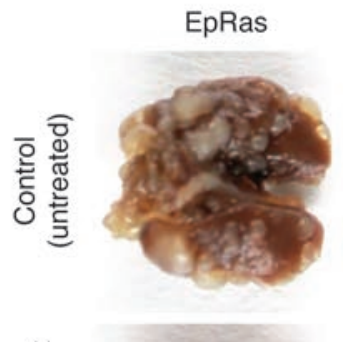

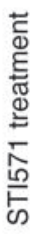

B Metastases (no. per lung)
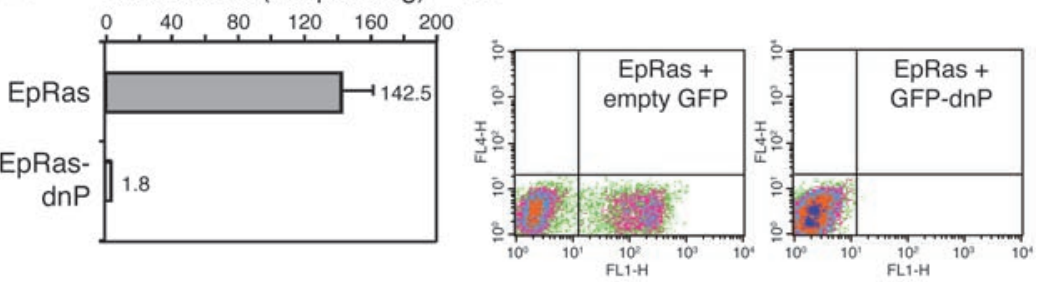

F

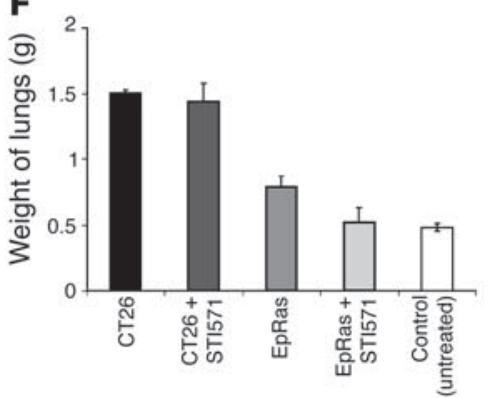

CT26 colon carc.
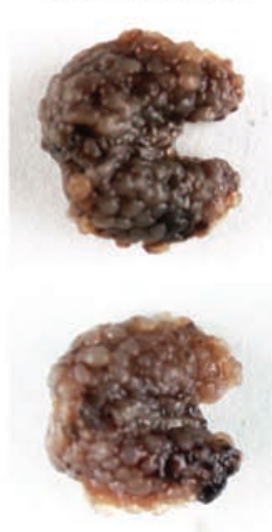

G

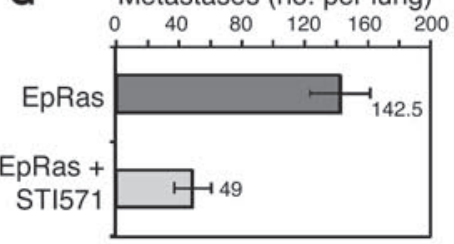

\section{Figure 7}

$\mathrm{dnP}$ and STI571 (Gleevec) prevent metastasis of EpRas cells in a cell-autonomous fashion. (A) EpRas cells expressing empty GFP vector (EpRas) or dnP (EpRas-dnP) were injected into the tail veins of nude mice $\left(5 \times 10^{5}\right.$ cells per mouse). Photographs of respective lungs are shown. (B) Mean numbers of lung metastases per lung (3 lungs per cell type) were quantitated by serial sectioning. (C and $\mathbf{D})$ Mixtures of EpRas cells (no GFP) and GFP-expressing EpRas ( $43 \%$ GFP ${ }^{+}$cells) or EpRas-GFP-dnP cells ( $37 \%$ GFP+ cells) were injected $\left(5 \times 10^{5}\right.$ cells) into the tail veins of nude mice. After 3 weeks, cells were recultivated from individual lungs and analyzed for GFP by FACS. One day after injection, 1 mouse per group was analyzed for injected cells initially reaching the lungs, yielding 8\% (EpRas/EpRas-GFP) and 12\% (EpRas/EpRas-GFP-dnP) GFP+ cells. (C) FACS profiles of lung cell cultures from representative mice of the 2 groups. (D) Percentage of GFP+ cells for individual mice. (E) Nude mice were treated or not treated for 6 days with STI571 and tail vein-injected with EpRas cells or CT26 cells $\left(5 \times 10^{5}\right.$ cells per animal) 1 day after start of STI571 treatment. All mice were sacrificed when controls were moribund ( $\sim 3$ weeks), and lungs were photographed. Lung metastases were quantified either by determining mean total lung weights from a total of 6-9 lungs each (F; mean weight \pm SD) or by serial sectioning to determine mean numbers of metastases per lung (G; mean \pm SD from 3 lungs total).

other than PI3K are activated by autocrine PDGFR signaling and required for maintenance of an invasive phenotype; (d) human carcinoma cell lines and samples from tumor patients confirm that autocrine PDGFR signaling may play a role in human breast cancer progression.

Autocrine PDGFR signaling during EMT: PI3K-dependent protection from TGF- $\beta$-induced apoptosis. During TGF- $\beta$-induced EMT of EpRas cells, an autocrine PDGF/PDGFR loop is established through upregulation of both ligands and receptors. This results in the expression of known target genes of PDGFR signaling and hyperactivation of PI3K. When attenuating PDGFR signaling with several agents including STI571 during EMT, PI3K pathway activity at basal levels was maintained. As a consequence, STI571-treated EpRas cells underwent apoptosis instead of EMT in response to TGF- $\beta$. Similar results were obtained with EpRas cells treated with the specific PI3K inhibitor LY294.002 (19). Therefore, induction of PDGF autocrine signaling at the onset of EMT cooperates with Ras to produce the high PI3K activity required to protect EpRas cells from TGF- $\beta$-induced apoptosis during EMT. In contrast, EpRasXT cells after EMT did not require an autocrine PDGF loop for survival, since PI3K inhibition by LY294.002 or blockade of PDGFR signaling by respective pharmacological inhibitors do not elicit apoptosis in EpRasXT cells.

Functional significance of other signaling pathways activated by autocrine PDGF signaling. Besides promoting PI3K-mediated survival during EMT, sustained autocrine PDGFR signaling contributed to maintenance of EMT. In response to 2 pharmacological PDGFR tyrosine kinase inhibitors, EpRasXT cells lost their typical spindle-shaped morphology, regained plasma membrane E-cadherin expression, and downregulated mesenchymal markers. Treatment of EpRasXT cells with the PI3K inhibitor LY294.002 elicited no comparable response, suggesting that the effect of PDGFR signaling on the mesenchymal phenotype might be due to other signaling pathways.

One such additional, EMT-specific pathway activated by autocrine PDGFR signaling involves STAT1. In EpH4-derived cells (EpC40XT) undergoing TGF- $\beta$-induced scattering (19), the basal levels of STAT1 expressed are not enhanced and activated by PDGF. In contrast, cells capable of undergoing EMT (EpRasXT and EpS35XT) express elevated levels of STAT1, which are fur- 
ther induced and activated by PDGF (Supplemental Figure 1). In line with this observation, numerous genes of the IFN- $\gamma /$ STAT1 signaling pathways were upregulated in an EMT-specific fashion (29). Therefore, PDGF-dependent STAT1 signaling might contribute to EMT, but respective mechanisms remain to be inves-

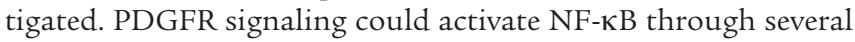
pathways in addition to PI3K signaling (50). It will be interesting to determine whether PDGFR-dependent activation of the NF-кB pathway could contribute to EMT, including activation of many EMT-specific genes, as recently described (51).

PDGFR signaling: cell-autonomous promotion of tumor cell metastasis. Previous work on the role of tumor cell-secreted PDGF in carcinoma progression and metastasis suggested that its main role was to elicit responses in the tumor stroma, in particular to cause tumor angiogenesis $(36,37,52,53)$. For instance, in colon carcinoma cells, TGF- $\beta$-induced PDGF was ascribed to act exclusively via paracrine effects on tumor vascularization (54). While paracrine action of PDGF on the tumor stroma is undoubtedly an important mechanism in tumor progression $(36,42,55)$, our studies provide evidence for an autocrine, cell-autonomous activity of PDGF during metastasis. We showed that in vivo interference with PDGFR signaling in EpRas cells injected into mice - by STI571 treatment or $\mathrm{dnP}$ overexpression - significantly reduced or even prevented lung metastasis. This appears to be a specific effect on the injected tumor cells, and not on other cells in the animal, as in the murine colon carcinoma cell line with a constitutive EMT phenotype (CT26; ref. 46), STI571 completely failed to affect metastasis formation. Rather than PDGFR signaling, CT26 cells might employ (STI571-insensitive) VEGFR signaling for survival during EMT and metastasis, similar to colonic organoids that express the VEGFR Flt1 and VEGF during EMT (56). When EMT was reversed in these cells by a dominant-negative TGF- $\beta$ RII (10), VEGFR rather than PDGFR was downregulated (29). In line with this, human colon carcinomas show enhanced VEGF production, while agents blocking VEGFR signaling attenuated tumor growth and metastasis in mice injected with CT26 cells (57). Accordingly CT26 cells sustain STI571 treatment in culture (Supplemental Figure 4).

Several findings support our notion that PDGFR inhibition abrogates metastasis formation of EpRas cells in a cell-autonomous fashion. First, a 5-day treatment with STI571 after tumor cell injection is unlikely to act via inhibition of vascularization, as the angiogenic switch only occurs after the initial tumor has exceeded a certain size. Second, STI571-mediated interference with PDGF effects on stromal or endothelial cells should have similarly inhibited metastasis formation by EpRas and CT26 cells. Most importantly, however, GFP-labeled EpRas-dnP cells failed to contribute to metastases formed by neighboring unlabeled EpRas cells. This renders a role of stromal or endothelial cells unlikely, since the EpRas cells could establish crosstalk to stromal/endothelial cells via multiple secreted factors such as chemokines (58).

The short duration of STI571 treatment able to attenuate metastasis formation by EpRas cells also makes it unlikely that inhibition of PDGFR signaling would cause a proliferation defect of the EpRas cells at sites of distant metastasis. If injected EpRas cells penetrate the vascular endothelium and survive as dormant cells in the lung during STI571 treatment, they should reinitiate proliferation when drug treatment is terminated. Such dormant cells should induce numbers of smaller metastatic lesions similar to those in control mice. Since we observed a strongly reduced number of metastatic foci in STI571-treated animals, which were of a size similar to those in control mice, we favor the idea that autocrine PDGF signaling functions during extravasation in a cell-autonomous fashion.

Cell-autonomous, autocrine PDGF signaling: relevance in mouse models and human breast cancer. Our results in the MMTV-Neu/HER2 transgenic mouse tumor model support that autocrine PDGF signaling contributes to tumor progression and metastasis in vivo. While mammary tumors from MMTV-Neu/HER2 transgenic mice showed no evidence for EMT in vivo, respective tumors from MMTV-Neu/HER $2 \times M M T V-T G F-\beta 1$ mice expressed the EMT markers vimentin and tenascin $C$ widely (Figure 2; ref. 29) and display enhanced PI3K activity (11). Importantly, in these tumors, PDGF and the PDGF target gene MCP-1 are upregulated as compared with tumors in MMTV-Neu mice, consistent with a TGF- $\beta$ induced autocrine PDGF loop.

Several lines of evidence also suggest a function of autocrine PDGF signaling in human breast cancer progression. First, STI571 and a more specific PDGFR tyrosine kinase inhibitor induced apoptosis or abolished the migratory phenotype in 2 human mammary carcinoma cell lines that exhibit a constitutive, mesenchymal EMT phenotype (47). Second, expression of both PDGFR $\alpha$ and PDGFR $\beta$ in human mammary carcinomas as detected by immunohistochemistry correlated with a malignant, invasive tumor phenotype, while normal breast tissue did not express these receptors. It was previously reported that elevated levels of PDGF correlate with unfavorable prognosis in human breast carcinomas (34). Increased PDGF expression was also found in late-stage breast cancer patients $(35,59)$. Finally, expression of both PDGF receptors $(\alpha$ and $\beta$ ) was detected in malignant breast cancer (60), pancreatic cancer, and pancreatic as well as prostate cancer cell lines $(61,62)$. In these cell lines, inhibition of PDGFR signaling with STI571 significantly reduced experimental metastasis in nude mice. In glioblastoma cell lines and mouse models, the importance of autocrine PDGFR signaling for cancer progression is already firmly established (33, 63-65).

STI571 as an antimetastatic drug. Besides its main application in therapy of chronic myelogenous leukemia via BCR-ABL inhibition, STI571 (66) is increasingly used in the treatment of other diseases, including those caused by hyperactivation of the PDGFR signaling pathway through gene fusion (67). Together with the clinical evidence by others $(34,35,59)$, our data provide a clear functional role for autocrine PDGFR signaling in the cell-autonomous acquisition and maintenance of the metastatic phenotype of mammary tumor cells. In addition to inhibition of angiogenesis induction by paracrine PDGF signaling, PDGFR inhibition by STI571 might also interfere with metastasis through cell-autonomous prevention of metastatic dissemination. Since STI571 can also inhibit tumor cell proliferation $(37,52)$, reactivation of dormant micrometastases might also be prevented. This would justify investigation of the potential efficacy of STI571 for mammary and other metastatic human tumors, which might rely on PDGFR signaling for tumor cell survival and dedifferentiation. As STI571 is an approved drug, it could also be included in clinical trials performed in combination with other breast cancer drugs, in the hope that it may reduce breast cancer metastasis.

\section{Methods}

Cells and cell culture. Origin and culture conditions for EpH4 mouse mammary epithelial cells, their Has-Ras transformed derivatives EpRas and EpRasXT, and CT26 colon carcinoma cells expressing or lacking dnTGF- $\beta$ RII 
were described previously $(10,23)$. Generation and culture conditions of V12-Ras-, S35-Ras-, C40-Ras-, and Bcl-2-expressing EpH4 cells are described in ref. 19. EpRas clones expressing $\mathrm{dnP}$ (45) were generated similarly. The human mammary carcinoma cell lines MDA-MB-231 and CAMA-1 (a kind gift of Andreas Eger, Medical University Vienna, Vienna, Austria) were cultivated and subjected to collagen gel culture as described in Supplemental Methods.

Collagen gel culture and marker analysis. Serum-free, 3D cultures of EpRas cells and their derivatives were described previously (19, 23). For description of inhibitors, growth factors, and antibodies applied, see Supplemental Methods. TUNEL assay and confocal immunofluorescence analysis was performed on 3D structures as described in ref. 19 and Supplemental Methods.

Assay for PDGF-dependent autocrine loop. Hu-PDGF- $\alpha$-R-expressing murine hematopoietic cells (huPDGFR/FDCP-1) - generated as EGFR/FDCP-1 cells (68) - were obtained from BI Austria and grown in StemPro-34 plus IL-3 (2 ng/ml; R\&D Systems). Confluent EpRas and EpRasXT cultures were incubated with StemPro-34 minus serum supplement (Invitrogen Corp.) plus bovine pituitary extract (concentration as used for collagen gel culture media), and respective supernatants were concentrated 10 -fold by ultrafiltration after 2 days. Proliferation of huPDGFR/FDCP-1 reporter cells in response to these supernatants was measured by $\left[{ }^{3} \mathrm{H}\right]$ thymidine uptake (68). As a specificity control, secreted PDGF in the above supernatants was neutralized with $\alpha$-PDGF-neutralizing antibody or PDGFR inhibitor. For standardization, a dilution series of purified human PDGF (0.1-10 ng/ml; R\&D Systems) was used to calculate the amount of bioactive PDGF corresponding to the specific $\left[{ }^{3} \mathrm{H}\right]$ thymidine incorporation (cpm EpRasXT minus cpm EpRasXT $+\alpha$-PDGF).

Western blot analysis. Cells were treated where indicated with PDGF (10 ng/ml), PDGF-neutralizing antibody $(20 \mu \mathrm{g} / \mathrm{ml})$, control nonimmune antibodies $(20 \mu \mathrm{g} / \mathrm{ml})$, PDGFR inhibitor $(2 \mu \mathrm{M})$, or STI571 (Gleevec; Novartis) $(1 \mu \mathrm{M})$ for 30 minutes. Lysates were collected and subjected to Western blot analysis performed as described previously (19). See Supplemental Methods for antibodies used.

EMSA. EMSAs for STAT1/STAT3 were performed as described previously (69). For supershift reactions of STAT-containing complexes, $0.2 \mu \mathrm{g}$ of antibodies to STAT1 (M22; Santa Cruz Biotechnology Inc.) and STAT3 (c-20; Santa Cruz Biotechnology Inc.) were employed (see Supplemental Methods for details on EMSA performance).

Tumorigenesis and metastasis assays. Athymic MF1 nude mice (6-10 weeks old) were used for mammary gland and tail vein injections as described previously (19). For details, see Supplemental Methods. All animal procedures performed were approved by the Institutional Animal Care and Use Committee of the Research Institute for Molecular Pathology and by the Bundesministerium fuer Bildung, Wissenschaft und Kultur (Vienna, Austria).

Tissue samples and immunohistochemistry. Breast cancer progression tissue microarrays included 16 normal mammary gland samples, 45 intraductal mammary carcinoma samples, and 93 invasive mammary carcinoma samples. For antibodies used and procedure details, see Supplemental Methods.
Transgenic mice. FVB/N-TgN(MMTVneu)202Mul (stock no. 002376) and FVB/NJ-Tg(MMTVTGFB1)46Hlm/J (stock no. 002933) mice were obtained from the Jackson Laboratory. MMTV-Neu/MMTV-TGF- $\beta 1$ and MMTV-Neu females were maintained separate from males until tumors developed. Three tumors originating from different mice were frozen in liquid nitrogen for each genotype, pulverized frozen using a microdismembrator (VWR), and RNA isolated using TRIzol (Invitrogen Corp.). Integrity and quantity of mRNA obtained was tested using a bioanalyzer (Agilent).

Quantitative RT-PCR. mRNA quantitation was performed using the TaqMan EZ RT-PCR kit (Applied Biosystems), and all samples were analyzed in triplicate on the ABI PRISM 7700 Sequence Detection System (Applied Biosystems). See Supplemental Methods for reagents used.

Statistics. Four independent experiments for each tumor were analyzed by 2 -tailed Student's $t$ tests to check for significant differences in expression levels for each gene tested.

\section{Acknowledgments}

We thank K. Politi and C. Summerfield for comments on the manuscript and G. Litos and I. Tamir for expert technical assistance, the Department of Biological Sciences, University of Iowa, for anti-cytokeratin antibodies (TROMA-I), and G. Loeber, Boehringer Ingelheim-Austria, for supplying us with huPDGFR/FDCP-1 cells and a specific PDGFR tyrosine kinase inhibitor. This work was supported by grants from a European Union Training and Mobility of Researchers Programme (ERBFMRXCT-980197), the Austrian Research funding agency (FWF; SFB 006/612 and SFB F28), and the Austrian Industrial Research Promotion Fund (FFF project no. 803776).

Received for publication February 4, 2005, and accepted in revised form March 21, 2006.

Address correspondence to: Hartmut Beug, Research Institute for Molecular Pathology, Dr. Bohrgasse 7, A-1030 Vienna, Austria. Phone: 43-1-79730-883; Fax: 43-1-7987153; E-mail: beug@ imp.univie.ac.at. Or to: Martin Jechlinger, Memorial Sloan-Kettering Cancer Center, 1225 York Avenue, New York, New York 10021, USA. Phone: (212) 639-6193; Fax: (212) 717-3125; E-mail: jechlinm@mskcc.org.

Martin Jechlinger's present address is: Memorial Sloan-Kettering Cancer Center, New York, New York, USA.

Richard Moriggl's present address is: Ludwig Boltzmann Institute for Cancer Research, Vienna, Austria.

Stefan Grünert's present address is: Children's Cancer Research Institute, Vienna, Austria.

Hartmut Beug and Stefan Grünert contributed equally to this work.
1. Hanahan, D., and Weinberg, R.A. 2000. The hallmarks of cancer. Cell. 100:57-70.

2. Hay, E.D. 1995. An overview of epitheliomesenchymal transformation. Acta Anat. (Basel). 154:8-20.

3. Grossmann, J. 2002. Molecular mechanisms of "detachment-induced apoptosis - anoikis." Apoptosis. 7:247-260.

4. Birchmeier, C., Birchmeier, W., and Brand-Saberi, B. 1996. Epithelial-mesenchymal transitions in cancer progression. Acta Anat. 156:217-226.

5. Boyer, B., Valles, A.M., and Edme, N. 2000. Induction and regulation of epithelial-mesenchymal transitions. Biochem. Pharmacol. 60:1091-1099.

6. Thiery, J.P. 2003. Epithelial-mesenchymal transitions in development and pathologies. Curr. Opin. Cell Biol. 15:740-746.

7. Akhurst, R.J., and Derynck, R. 2001. TGF-beta signaling in cancer - a double-edged sword. Trends Cell Biol. 11:S44-S51.

8. Derynck, R., Akhurst, R.J., and Balmain, A. 2002. TGF-beta signaling in tumor suppression and cancer progression. Nat. Genet. 29:117-129.

9. Cui, W., et al. 1996. TGFbeta1 inhibits the formation of benign skin tumors, but enhances progression to invasive spindle carcinomas in transgenic mice.
Cell. 86:531-542.

10. Oft, M., Heider, K., and Beug, H. 1998. TGFbeta signaling is necessary for carcinoma cell invasiveness and metastasis. Curr. Biol. 8:1243-1252.

11. Muraoka, R.S., et al. 2003. Increased malignancy of Neu-induced mammary tumors overexpressing active transforming growth factor beta1. Mol. Cell. Biol. 23:8691-8703.

12. Yang, Y.A., et al. 2002. Lifetime exposure to a soluble TGF- $\beta$ antagonist protects mice against metastasis without adverse side effects. J. Clin. Invest. 109:1607-1615. doi:10.1172/JCI200215333.

13. Weeks, B.H., He, W., Olson, K.L., and Wang, X.J. 
2001. Inducible expression of transforming growth factor beta 1 in papillomas causes rapid metastasis. Cancer Res. 61:7435-7443.

14. Jacob, S., and Praz, F. 2002. DNA mismatch repair defects: role in colorectal carcinogenesis. Biochimie. 84:27-47.

15. Degen, W.G., et al. 1996. Expression of nma, a novel gene, inversely correlates with the metastatic potential of human melanoma cell lines and xenografts. Int. J. Cancer. 65:460-465.

16. Markowitz, S., et al. 1995. Inactivation of the type II TGF-beta receptor in colon cancer cells with microsatellite instability. Science. 268:1336-1338.

17. Schutte, M., et al. 1996. DPC4 gene in various tumor types. Cancer Res. 56:2527-2530.

18. Linardopoulos, S., et al. 1995. Deletion and altered regulation of $\mathrm{p} 16 \mathrm{INK} 4 \mathrm{~A}$ and $\mathrm{p} 15 \mathrm{INK} 4 \mathrm{~B}$ in undifferentiated mouse skin tumors. Cancer Res. 55:5168-5172.

19. Janda, E., et al. 2002. Ras and TGFbeta cooperatively regulate epithelial cell plasticity and metastasis: dissection of Ras signaling pathways. J. Cell Biol. 156:299-313

20. Nahta, R., Hortobagyi, G.N., and Esteva, F.J. 2003. Growth factor receptors in breast cancer: potential for therapeutic intervention. Oncologist. 8:5-17.

21. Bos, J.L. 1989. ras oncogenes in human cancer: a review. Cancer Res. 49:4682-4689.

22. Rajagopalan, H., et al. 2002. Tumorigenesis: RAF/ RAS oncogenes and mismatch-repair status. Nature. 418:934.

23. Oft, M., et al. 1996. TGF-beta1 and Ha-Ras collaborate in modulating the phenotypic plasticity and invasiveness of epithelial tumor cells. Genes Dev. 10:2462-2477.

24. Lehmann, K., et al. 2000. Raf induces TGFbeta production while blocking its apoptotic but not invasive responses: a mechanism leading to increased malignancy in epithelial cells. Genes Dev. 14:2610-2622.

25. Gotzmann, J., et al. 2002. Hepatocytes convert to a fibroblastoid phenotype through the cooperation of TGF-beta 1 and Ha-Ras: steps towards invasiveness. J. Cell Sci. 115:1189-1202.

26. Bates, R.C., and Mercurio, A.M. 2003. Tumor necrosis factor-alpha stimulates the epithelial-tomesenchymal transition of human colonic organoids. Mol. Biol. Cell. 14:1790-1800.

27. Jechlinger, M., Grunert, S., and Beug, H. 2002. Mechanisms in epithelial plasticity and metastasis: insights from 3D cultures and expression profiling. J. Mammary Gland Biol. Neoplasia. 7:415-432.

28. Grunert, S., Jechlinger, M., and Beug, H. 2003. Diverse cellular and molecular mechanisms contribute to epithelial plasticity and metastasis. Nat. Rev. Mol. Cell Biol. 4:657-665.

29. Jechlinger, M., et al. 2003. Expression profiling of epithelial plasticity in tumor progression. Oncogene. 22:7155-7169.

30. Huber, M.A., Kraut, N., and Beug, H. 2005. Molecular requirements for epithelial-mesenchymal transition during tumor progression. Curr. Opin. Cell Biol. 17:1-11.

31. Kang, Y., and Massague, J. 2004. Epithelialmesenchymal transitions: twist in development and metastasis. Cell. 118:277-279.

32. Brabletz, T., Jung, A., Spaderna, S., Hlubek, F., and Kirchner, T. 2005. Migrating cancer stem cells - an integrated concept of malignant tumour progression. Nat. Rev. Cancer. 5:744-749.

33. Dai, C., et al. 2001. PDGF autocrine stimulation dedifferentiates cultured astrocytes and induces oligodendrogliomas and oligoastrocytomas from neural progenitors and astrocytes in vivo. Genes Dev. 15:1913-1925.

34. Seymour, L., and Bezwoda, W.R. 1994. Positive immunostaining for platelet derived growth factor (PDGF) is an adverse prognostic factor in patients with advanced breast cancer. Breast Cancer Res. Treat. 32:229-233.

35. Ariad, S., Seymour, L., and Bezwoda, W.R. 1991. Platelet-derived growth factor (PDGF) in plasma of breast cancer patients: correlation with stage and rate of progression. Breast Cancer Res. Treat. 20:11-17.

36. Pietras, K., Sjoblom, T., Rubin, K., Heldin, C.H., and Ostman, A. 2003. PDGF receptors as cancer drug targets. Cancer Cell. 3:439-443.

37. Uehara, H., et al. 2003. Effects of blocking platelet-derived growth factor-receptor signaling in a mouse model of experimental prostate cancer bone metastases. J. Natl. Cancer Inst. 95:458-470.

38. Klinghoffer, R.A., Hamilton, T.G., Hoch, R., and Soriano, P. 2002. An allelic series at the PDGFalphaR locus indicates unequal contributions of distinct signaling pathways during development. Dev. Cell. 2:103-113.

39. Heldin, C.H., and Westermark, B. 1999. Mechanism of action and in vivo role of platelet-derived growth factor. Physiol. Rev. 79:1283-1316.

40. Muller, W.J., Sinn, E., Pattengale, P.K., Wallace, R., and Leder, P. 1988. Single-step induction of mammary adenocarcinoma in transgenic mice bearing the activated c-neu oncogene. Cell. 54:105-115.

41. Siegel, P.M., Shu, W., Cardiff, R.D., Muller, W.J., and Massague, J. 2003. Transforming growth factor beta signaling impairs Neu-induced mammary tumorigenesis while promoting pulmonary metastasis. Proc. Natl. Acad. Sci. U. S. A. 100:8430-8435.

42. Buchdunger, E., O'Reilly, T., and Wood, J. 2002. Pharmacology of imatinib (STI571). Eur. J. Cancer. 38(Suppl. 5):S28-S36

43. Buettner, R., Mora, L.B., and Jove, R. 2002. Activated STAT signaling in human tumors provides novel molecular targets for therapeutic intervention. Clin. Cancer Res. 8:945-954.

44. Janda, E., Litos, G., Grunert, S., Downward, J. and Beug, H. 2002. Oncogenic Ras/Her-2 mediate hyperproliferation of polarized epithelial cells in $3 \mathrm{D}$ cultures and rapid tumor growth via the PI3K pathway. Oncogene. 21:5148-5159.

45. Ueno, H., Escobedo, J.A., and Williams, L.T. 1993. Dominant-negative mutations of platelet-derived growth factor (PDGF) receptors. Inhibition of receptor function by ligand-dependent formation of heterodimers between PDGF alpha- and betareceptors. J. Biol. Chem. 268:22814-22819.

46. Brattain, M.G., Strobel-Stevens, J., Fine, D., Webb, M., and Sarrif, A.M. 1980. Establishment of mouse colonic carcinoma cell lines with different metastatic properties. Cancer Res. 40:2142-2146.

47. Eger, A., et al. 2005. DeltaEF1 is a transcriptional repressor of E-cadherin and regulates epithelial plasticity in breast cancer cells. Oncogene. 24:2375-2385

48. De Caestecker, M.P., Piek, E., and Roberts, A.B. 2000. Role of transforming growth factor-beta signaling in cancer. J. Natl. Cancer Inst. 92:1388-1402.

49. Dumont, N., and Arteaga, C.L. 2000. Transforming growth factor-beta and breast cancer: tumor promoting effects of transforming growth factor-beta. Breast Cancer Res. 2:125-132.

50. Huber, M.A., Beug, H., and Wirth, T. 2004. Epithelial-mesenchymal transition: NF-kappaB takes center stage. Cell Cycle. 3:1477-1480.

51. Huber, M.A., et al. 2004. NF-кB is essential for epithelial-mesenchymal transition and metastasis in a model of breast cancer progression. J. Clin. Invest. 114:569-581. doi:10.1172/JCI200421358.

52. Yi, B., Williams, P.J., Niewolna, M., Wang, Y., and Yoneda, T. 2002. Tumor-derived platelet-derived growth factor-BB plays a critical role in osteosclerotic bone metastasis in an animal model of human breast cancer. Cancer Res. 62:917-923.

53. Cao, R., et al. 2004. PDGF-BB induces intratumoral lymphangiogenesis and promotes lymphatic metastasis. Cancer Cell. 6:333-345.

54. Hsu, S., Huang, F., and Friedman, E. 1995. Plateletderived growth factor-B increases colon cancer cell growth in vivo by a paracrine effect. J. Cell. Physiol. 165:239-245.

55. Bergers, G., Song, S., Meyer-Morse, N., Bergsland, E., and Hanahan, D. 2003. Benefits of targeting both pericytes and endothelial cells in the tumor vasculature with kinase inhibitors. J. Clin. Invest. 111:1287-1295. doi:10.1172/JCI200317929.

56. Bates, R.C., et al. 2003. Flt-1-dependent survival characterizes the epithelial-mesenchymal transition of colonic organoids. Curr. Biol. 13:1721-1727.

57. Shaheen, R.M., et al. 2001. Effects of an antibody to vascular endothelial growth factor receptor-2 on survival, tumor vascularity, and apoptosis in a murine model of colon carcinomatosis. Int.J. Oncol. 18:221-226.

58. Muller, A., et al. 2001. Involvement of chemokine receptors in breast cancer metastasis. Nature. 410:50-56.

59. Anan, K., et al. 1996. Vascular endothelial growth factor and platelet-derived growth factor are potential angiogenic and metastatic factors in human breast cancer. Surgery. 119:333-339.

60. Coltrera, M.D., Wang, J., Porter, P.L., and Gown, A.M. 1995. Expression of platelet-derived growth factor B-chain and the platelet-derived growth factor receptor beta subunit in human breast tissue and breast carcinoma. Cancer Res. 55:2703-2708.

61. Kim, S.J., et al. 2004. Simultaneous blockade of platelet-derived growth factor-receptor and epidermal growth factor-receptor signaling and systemic administration of paclitaxel as therapy for human prostate cancer metastasis in bone of nude mice. Cancer Res. 64:4201-4208.

62. Hwang, R.F., et al. 2003. Inhibition of plateletderived growth factor receptor phosphorylation by STI571 (Gleevec) reduces growth and metastasis of human pancreatic carcinoma in an orthotopic nude mouse model. Clin. Cancer Res. 9:6534-6544.

63. Shamah, S.M Stiles, C.D and Guha, A. 1993. Dominant-negative mutants of platelet-derived growth factor revert the transformed phenotype of human astrocytoma cells. Mol. Cell. Biol. 13:7203-7212.

64. Vassbotn, F.S., et al. 1994. Activated platelet-derived growth factor autocrine pathway drives the transformed phenotype of a human glioblastoma cell line. J. Cell. Physiol. 158:381-389.

65. Liu, Y.C., Chen, S.C., Chang, C., Leu, C.M., and $\mathrm{Hu}$, C.P. 1996. Platelet-derived growth factor is an autocrine stimulator for the growth and survival of human esophageal carcinoma cell lines. Exp. Cell Res. 228:206-211.

66. Capdeville, R., Buchdunger, E., Zimmermann, J., and Matter, A. 2002. Glivec (STI571, imatinib), a rationally developed, targeted anticancer drug. Nat. Rev. Drug Discov. 1:493-502.

67. Apperley, J.F., et al. 2002. Response to imatinib mesylate in patients with chronic myeloproliferative diseases with rearrangements of the plateletderived growth factor receptor beta. N. Engl. J. Med. 347:481-487.

68. Von Ruden, T., and Wagner, E.F. 1988. Expression of functional human EGF receptor on murine bone marrow cells. EMBO J. 7:2749-2756.

69. Moriggl, R., et al. 1998. Activation of STAT proteins and cytokine genes in human Th1 and Th2 cells generated in the absence of IL-12 and IL-4. J. Immunol. 160:3385-3392. 